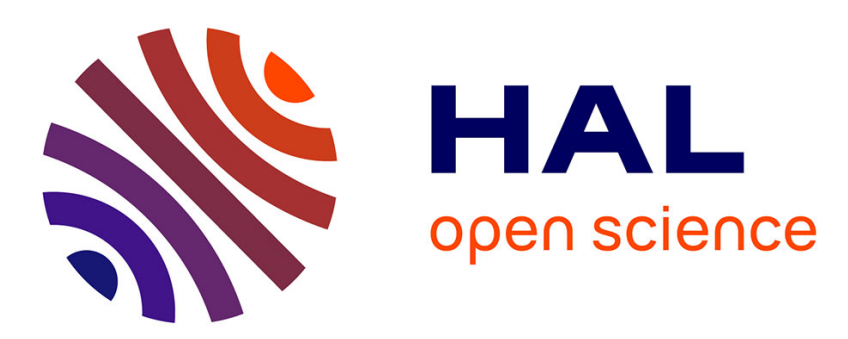

\title{
L'introduction des référents dans le discours en français : contraintes cognitives et développement des compétences narratives.
}

\author{
Monique Vion, Annie Colas
}

\section{- To cite this version:}

Monique Vion, Annie Colas. L'introduction des référents dans le discours en français: contraintes cognitives et développement des compétences narratives.. 1999, pp.383-387. hal-00134173

\author{
HAL Id: hal-00134173 \\ https://hal.science/hal-00134173
}

Submitted on 1 Mar 2007

HAL is a multi-disciplinary open access archive for the deposit and dissemination of scientific research documents, whether they are published or not. The documents may come from teaching and research institutions in France or abroad, or from public or private research centers.
L'archive ouverte pluridisciplinaire HAL, est destinée au dépôt et à la diffusion de documents scientifiques de niveau recherche, publiés ou non, émanant des établissements d'enseignement et de recherche français ou étrangers, des laboratoires publics ou privés. 


\title{
L'introduction des référents dans le discours en français : contraintes cognitives et développement des compétences narratives
}

Introducing referents in French: cognitive constraints and development of narrative skills.

\author{
Monique Vion et Annie Colas \\ CREPCO UMR 6561 du CNRS, \\ Université de Provence, \\ 29 , av. Robert Schuman, \\ F-13621 Aix en Provence cedex 1
}

Le recueil d'observations fondé sur des images tient une place importante dans les recherches développementales sur l'introduction, le maintien et le rappel de la référence dans le discours. Or le traitement des images implique des activités cognitives (inférentielles et mnémoniques) encore mal connues et qui demande à être analysé pour lui même. En effet les connaissances sur la gestion de la référence dans le discours proviennent indifféremment d'études où la verbalisation débute alors qu'un contenu d'ensemble a été élaboré ou bien où celle-ci est effectuée au fur et à mesure de l'acquisition du contenu. Nous avons observé que la production change selon les informations disponibles au moment de la verbalisation (Canoz et Vion, 1994). Par ailleurs, Trabasso et ses collaborateurs ont souligné l'importance de l'activité d'inférence dans la verbalisation de séries d'images (Trabasso, Stein, Rodkin, Munger et Baugh, 1992 ; Trabasso et Rodkin, 1994).

Selon nous, la manifestation des compétences linguistiques dans ce paradigme est tributaire à la fois de la disponibilité des informations à verbaliser et des inférences effectuées, et nos connaissances gagneraient en précision, si ces dimensions faisaient l'objet d'un contrôle expérimental. Nous avons fait varier la disponibilité des informations ainsi que la cohérence interne de séries d'images. Une obligation explicite était posée aux locuteurs : 1) il s'agit d'histoires, 2) elles sont racontables et pour ce faire, une mise en intrigue est nécessaire (Hausendorf, 1993). Nous avons étudié comment ils mentionnaient pour la première fois les personnages dans leur discours.

\section{METHODE}

Deux cent cinquante cinq garçons et filles, enfants (7,9 et 11 ans) et adultes, ayant le français pour langue native, ont participé au recueil. La passation individuelle durait 20 minutes.

Le locuteur était invité à raconter des bandes dessinées sans texte à quelqu'un qui en ignorait le contenu. Il devait les relater en tenant compte de chaque image, mais en évitant de donner trop de détails. Le destinataire (un pair d'âge de même niveau scolaire) était suffisament éloigné pour ne 
pas les voir. Il devait être attentif et écouter pour bien comprendre, sans parler. Il n'était auditeur qu'une seule fois au cours du recueil.

La première image de chaque bande dessinée (8 images) comportait deux personnages. Les autres n'en comportaient qu'un seul engagé dans diverses activités. Un lien minimal entre les images était assuré par la présence de l'un ou de l'autre des personnages de la première image. Les événements figurés de la deuxième à la dernière image impliquaient le même personnage quand le thème était maintenu. Quand il était changé, l'autre personnage de la première image était impliqué dans l'événement figuré sur la dernière image. De plus la disposition (gauche - droite) des personnages dans la première image était contrabalancée. Le "degré" d'inférence nécessaire pour élaborer une représentation d'ensemble de l'histoire a été manipulé en contrastant les bandes dessinées selon le caractère plus ou moins explicité par les images de leur enchaînement. Pour un enchaînement implicite, les événements, bien qu'organisés en séquence, étaient interchangeables du point de vue de leur chronologie. Pour un enchaînement explicite, ils obéissaient à un ordre chronologique plus contraint, sans pour autant offrir la rigidité de l'organisation séquentielle d'un script. L'encodage du contenu a été manipulé en opposant deux modes de présentation. Dans un cas toutes les images étaient disposées sur une seule page. Le locuteur en prenait connaissance dans le but de raconter immédiatement après. Dans l'autre cas, chacunes des images figurait sur une page d'un carnet. Le locuteur devait tourner les pages et raconter au fur et à mesure de la découverte des images.

Un locuteur a été confronté à l'un ou l'autre mode de présentation, à l'un ou à l'autre type d'enchaînement et aux deux types d'organisation thématique. Au cours de la passation chacun voyait huit bandes dessinées test et trois bandes dessinées de remplissage.

L'introduction des personnages doit tenir compte du caractère totalement nouveau de l'information pour l'interlocuteur. L'introduction peut être conjointe ("deux enfants", "un couple", "des amis", etc.), ou bien individualisée. Si les personnages sont introduits individuellement, ils peuvent l'être au moyen d'expressions indéfinies ("un petit garçon"), de noms propres ("Martine") ou bien encore de noms sans articles ("papa", "grand-mère"). Ceci devraît être mieux maîtrisé par les plus grands que par les plus petits, plus coutumiers du renvoi externe au discours. Les plus jeunes pourraient mentionner les personnages de façon inappropriée en employant des expressions définies à caractère déictique ("le petit garçon"), liées à la perception des images ou bien encore générique ("le papa"), liées à l'identification des personnages et à leur dénomination.

Ces attentes valent pour la situation où les locuteurs découvrent les images les unes après les autres (présentation consécutive des images). Mais le fait de prendre connaissance de l'ensemble des images (présentation globale), de pouvoir donc structurer au préalable une histoire et d'en planifier la verbalisation, devrait placer les locuteurs dans une situation optimale pour exercer leur 
capacité référentielle sur le mode intra-linguistique. Ceci devrait accentuer la différence de performance entre les divers groupes d'âge. Pour les enfants les plus âgés et les adultes, la facilitation de l'appréhension et/ou de l'établissement de liens entre les événements devrait conduire à un marquage initial de la cohésion d'ensemble du discours, plus prononcé que pour l'autre mode de présentation. Alors que, malgré un mode de présentation plus favorable à l'élaboration d'une narration et les injonctions de la consigne, la tâche devrait demeurer pour les enfants les plus jeunes, une tâche de dénomination. L'apphéhension en présentation globale du caractère plus ou moins explicite de l'enchaînement des images et de l'organisation thématique devrait également moduler les emplois.

\section{RESULTATS}

Les narrations enregistrées ont été transcrites selon les conventions de Hickmann, Liang, Hendricks et Roland (1990). Pour la partie de la production associée à la première image, la première mention des personnages a été codée ainsi : 1) "omission" : seul l'un des deux personnages est mentionné ; 2) "joint." : les personnages font l'objet d'une mention conjointe ("un couple ...", "deux amis ..", etc.) ; 3) les personnages sont introduits individuellement, de façon similaire et adéquate : "2IA" (deux articles indéfinis : "une dame et un monsieur" ; "2PN" (deux noms propres : "Pierre et Marie") ; "2GN"(deux noms génériques : "papa et maman") ou bien inadéquate : "2DA" (deux articles définis :"le monsieur et la dame") ; 4) les personnages sont introduits individuellement mais un possessif lie le second personnage mentionné au premier ; l'ensemble peut être adéquat : "IA+pos" (un article indéfini et un adjectif possessif : "un papa et son fils") ou bien inadéquat : "DA+pos" (un article défini et un adjectif possessif : "la grand mère et sa petite fille") ; 5) enfin les personnages sont marqués différemment : ou bien le premier personnage est marqué de façon inadéquate : "le singe i trouve un lion" ; "E.T. faisa ... sait une promenade sur le dos d'un lion" (un défini à gauche, "LD"); ou bien c'est l'inverse : "un monsieur et la dame" (un défini à droite, "RD"). Une fois le codage effectué, les omissions et les rares introductions par des noms propres ou sans articles (6\% des productions) ont été regroupées dans une seule catégorie : "autre". Les cas où les personnages ont été marqués différemment (LD : 4\% et RD : 3\%) ainsi que les introductions DA+pos (2\%) ont été aussi rangés dans la catégorie "autre". Celle-ci rassemble au total $15 \%$ des introductions, qui pour la plupart sont inadéquates. L'analyse a porté sur l'introduction inappropriée 2DA et les trois introductions appropriées : joint, 2IA et IA+pos qui rendent compte à elles seules de $76 \%$ des productions recueillies.

Les analyses de la variance effectuées ont porté sur le nombre d'occurrences d'un de ces types d'introduction. Les résultats commentés ci-dessous correspondent à des effets significatifs à des seuils inférieurs ou égaux à .05 . 


\section{Globalement}

D'une part, le mode de présentation influence l'emploi de l'introduction liée des personnages, qui est plus fréquente quand la bande dessinée est appréhendée d'emblée dans sa totalité. D'autre part, il influence l'emploi de la double introduction définie, qui est plus fréquente en présentation consécutive.

\section{en présentation consécutive (figure1)}

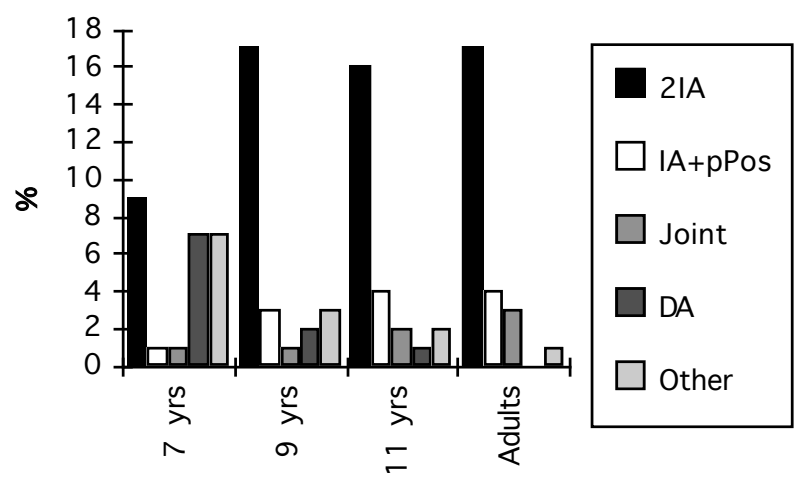

Figure 1 : Présentation consécutive : distribution des introductions en fonction de l'âge.

Consecutive presentation: distribution of introducing devices a function of age.

Le plus souvent, les locuteurs introduisent individuellement chacun des personnages comme des entités nouvelles par des expressions indéfinies. Conformément aux attentes, les expressions définies restent très importantes chez les $\mathrm{V}$ alors qu'elles sont très rares chez les adultes. L'introduction qui lie la présentation du second personnage mentionné au premier (IA+pos), rare à 7 ans, augmente régulièrement jusqu'à 11 ans et demeure stable au delà. L'introduction conjointe est principalement le fait des adultes.

\section{en présentation globale (figure 2)}

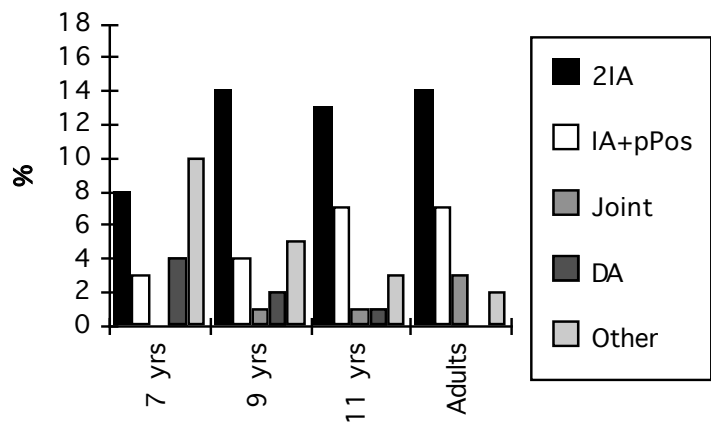

Implicit

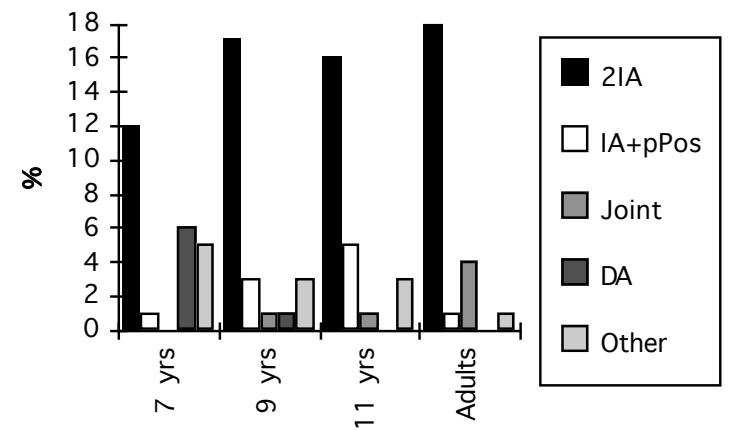

Explicit

Figure 2 : Présentation globale : distribution des introductions en fonction de l'âge et du type d'enchaînement.

Simultaneous presentation: distribution of introducing devices as a function of age. and type of linkage. 
L'emploi privilégié de l'introduction conjointe par les adultes se retrouve également ici, mais il est deux fois plus fréquent pour un enchaînement explicite. L'emploi en fonction de l'âge de la double introduction indéfinie est similaire à celui décrit en présentation consécutive, mais il s'accroit pour un enchaînement explicite. Il en va de même quand le thème change. Le nombre d'introductions individualisées indéfinies et liées varie en fonction de l'âge, du type d'enchaînement et de l'interaction entre ces deux facteurs. Pour un enchaînement implicite qui sucite globalement plus d'introduction de ce type qu'un enchaînement explicite, les emplois augmentent régulièrement chez les enfants et culminent à 11 ans. Ils régressent au dessous de la performance des enfants de 11 ans chez les adultes (qui lui substituent une introduction conjointe). Pour un enchaînement explicite, la hiérarchie demeure la même pour les enfants. En revanche, la performance des adultes se situe au niveau de celle des enfants de 7 ans (dans ce cas les adultes procèdent encore davantage par introduction conjointe).

\section{CONCLUSION}

Conformément aux attentes, par l'emploi dominant d'expressions définies diverses (" papa et maman " ; " le monsieur et la dame " ; " la grand mère et sa petite fille "), les enfants de 7 ans se sont avérés encore très centrés sur l'ici et le maintenant du commentaire des images. Alors que les plus âgés ont introduit les personnages en tenant compte du caractère nouveau de l'information pour le destinataire. Ces derniers ont présenté les personnages surtout au moyen d'une double introduction indéfinie (" une dame et un monsieur "). Ces résultats confortent les connaissances acquises dans diverses langues (Hickmann, 1995) et en particulier, celles concernant le français (Karmiloff-Smith, 1985 ; Hickmann, Kail et Roland, 1995). Ils permettent aussi de préciser les changements développementaux. Quand les locuteurs se sont placés dans le cadre de la narration, ils ont aussi employé des moyens qui, en plus de la nouveauté de l'information, indiquent un lien entre les personnages. L'introduction par un indéfini et un possessif ("un papa et son fils") distingue d'une part les enfants de 11 ans des deux autres groupes d'enfants (qui marquent très peu de tels liens) et d'autre part des adultes (qui emploient l'introduction conjointe : " un couple ", " deux amis "). Les enfants de 9 ans, tout en se montrant soucieux et capables d'exprimer la nouveauté de l'information, marquent moins explicitement que leurs aînés les relations entre les personnages que l'histoire va mettre en scène. Ce résultat rejoint celui de Trabasso et Rodkin (1994) qui observent en anglais un marquage accru de la possession avec l'âge.

Conformément aux attentes également, la présentation sous forme de carnet favorise une appréhension des personnages de la première image (seule connue du locuteur au moment où débute la narration) comme un tout à décrire ici et maintenant, indépendament des images subséquentes, alors que la présentation sur une seule page oblige davantage le locuteur, s'il en a la 
compétence, à s'engager dans une narration. Celle-ci dépend alors surtout du type d'enchaînement. Confrontés à un enchaînement explicite ils ont géré l'introduction de l'information nouvelle au moyen des expressions conventionnellement prévues dans la langue à cet effet. Tandis que confrontés à des événements n'ayant pas de liens très apparents, ils ont fait des inférences et mentionné dès le début de leur narration les relations qu'ils supposaient entre les personnages.

La manipulation expérimentale des contraintes inhérentes au traitement du support imagé s'avère donc un bon moyen d'évaluer la nature des changements développementaux. Elle doit permettre à l'avenir de moduler les connaissances antérieurement acquises. Elle doit aussi permettre de mieux comprendre en quoi certains contextes de la vie quotidienne sont plus favorables que d'autres à la manifestation des compétences référentielles et narratives des locuteurs.

\section{REFERENCES}

Berman, R. , \& Slobin, D. (Eds.)1994. Relating events in narrative: a crosslinguistic developmental study, Hillsdale, N.J., Erlbaum, 748p..

Canoz, F., \& Vion, M. 1994. Encoding and maintaining reference in oral discourse. International Journal of Psychology, 29 (5), 537-564.

Hausendorf, H. 1993. L'adulte auditeur, aspects de l'étayage dans l'interaction avec des narrateurs enfants. Acquisition et Interaction en Langue Etrangère, 2, 85-105.

Hickmann, M. 1995. Discourse organization and the development of reference to person, space and time, in P. Fletcher \& B. MacWhinney (Eds.). Handbook of Child Language., Oxford, Blakwell Publishers, 194-218.

Hickmann, M., Kail, M., \& Roland, F. 1995. Organisation référentielle dans les récits d'enfants en fonction des contraintes contextuelles. Enfance, 2, 215-226.

Hickmann, M., Liang, J., Hendricks, H., \& Roland, F. 1990. The development of discourse cohesion, coding manual, Nijmegen, Max Planck Institute for Psycholinguistics, 146p.

Karmiloff-Smith, A. 1985. Language and cognitive processes from a developmental perspective. Language and Cognitive Processes, 11, 61-85.

Trabasso, T, \& Rodkin, P. 1994. Knowledge of goals/plans, A conceptual basis for narrating "Frog where are you?", in R. Bernan \& D. Slobin (Eds.). Relating events in narrative: A crosslinguistic developmental study, Hillsdale, NJ , Erlbaum, 85-106.

Trabasso, T., Stein, N., Rodkin, P., Munger, M., \& Baughn, C. 1992. Knowledge of goals and plans in the on-line narration of events. Cognitive Development, 7, 133-190. 